\title{
The media and cancer survival
}

\author{
Heather Goodare
}

$J$ R Soc Med 2007;100:483-484

Surviving cancer presents several challenges: coping with the side effects of treatment, dodging the sword of Damocles, and decoding cancer stories in the media are just some of them.

' "Critical error" in cancer patient's treatment' was a recent headline in the national papers. The story of Lisa Norris, the schoolgirl who received 19 radiotherapy overdoses for her brain tumour at the Beatson Oncology Centre in Glasgow and died a year later, is no doubt a rare occurrence, and can be attributed to the errors of an 'under-qualified and undertrained treatment planner'. Unfortunately such stories are by no means unprecedented. Members of Radiotherapy Action Group Exposure (RAGE), an organization of breast cancer survivors founded in 1991, can also tell stories of deaths and second cancers attributed to the long-term effects of radiotherapy. ${ }^{1}$

Surely such serious problems arise from treatment errors, not standard treatment, and techniques are better now than they were 20 years ago? But high-dose treatment such as many of the RAGE women underwent is still regarded as 'standard', and administered not just occasionally or off-protocol but in the context of contemporary clinical trials. One arm of a current breast cancer trial, START Trial A, ${ }^{2}$ still includes infrequent highdose post-operative radiotherapy, which is more convenient for patients in the short term, and less costly for treatment centres. But is it really cost-effective in the long term?

There is a further problem. Media reports sometimes give a misleading impression of research. The publicity surrounding the publication in June 2006 of the fast-tracked article reporting the effect of radiotherapy fraction size and timing on breast cancer control ${ }^{2}$ provides an example. The press release from Cancer Research UK, ${ }^{3}$ with its emphatic headline 'Concentrated doses of radiotherapy shown to be better in treating breast cancer', stated: 'an experimental schedule of 13 larger doses . . . appears to offer the same protection against cancer returning in the same breast as the international standard of 25 smaller doses without any increase in side effects' [my emphasis]. This alleged result was widely reported, but is not borne out by the actual research (which will be superseded by the yet-unpublished START

3 Glengyle Terrace, Edinburgh EH3 9LL

Email: hm.goodare@virgin.net
Trial). This study should be taken together with the companion study on late adverse effects, ${ }^{4}$ which shows that the 'concentrated doses' have much worse side effects than the comparative regimes. To simplify the issue, how much long-term radiotherapy damage can be acceptable as a trade-off for lowering the risk of recurrence?

Patients are rarely told that radiotherapy to the breast can only improve local control; it cannot be claimed to extend overall survival, and long-term side effects include cardiac and lung damage. In spite of recent improvements in technique, in 1994 'a numerical difference in favour of non-irradiated patients' remained. ${ }^{5}$ Follow-up from newer trials looking at overall survival is still 'not sufficient to allow definitive conclusions to be drawn' ${ }^{6}$ It is therefore important to use fractionation schedules that minimize adverse effects, especially when there is increased use of cardiotoxic chemotherapy. Local recurrence is not pleasant, but patients and doctors need accurate information on which to base decisions. ${ }^{7}$

Patients can only make their own assessments of harms versus benefit if they are given the facts: this goes for drug treatments too. It has taken me twenty years to achieve recognition of one side effect of tamoxifen, which I found affected the singing voice. ${ }^{8}$ It is moreover unethical to publicize reports that do not give the full information needed by both patients and doctors, and may seriously mislead managers. People who draft medical press releases bear a heavy responsibility - busy journalists don't have time to study the papers on which they are based, for their target is tomorrow's deadline.

Plus ça change, plus c'est la même chose. Readers may remember the discredited study of women attending the Bristol Cancer Help Centre, published in 1990, ${ }^{9}$ which was widely publicized at the time with headlines such as: 'Cancer patients at holistic centre "are more likely to die". .10 In this instance again the damage was done, not by the paper in the Lancet, but by the press release issued by the CRC and the ICRF (now Cancer Research UK), who held a large press conference resulting in massive media coverage. Here too the press release ${ }^{11}$ went beyond the cautious conclusions of the paper, which itself was only an interim report of the proposed five-year study. The research was aborted because of the campaign by the Bristol Survey Support Group, comprising women who had taken part in the study and had lost confidence in the research team. ${ }^{12}$ The Bristol Cancer 
Help Centre nearly had to close after people lost faith in their expertise. Happily the Centre made a recovery from this setback after the Charity Commission reprimanded the two cancer charities for their part in the affair: it has subsequently gone from strength to strength, and now, renamed as Penny Brohn Cancer Care, is acknowledged to provide the gold standard in complementary cancer therapy.

The Bristol study was reported on after only half the proposed study period had elapsed. The same thing happened with the 'anastrozole alone or in combination with tamoxifen' (ATAC) trial, ${ }^{13}$ leading to sensational headlines such as 'New drug prevents breast cancer' at a time when such conclusions could not possibly have been drawn. Early reporting of trials that make inflated claims can only mislead. Researchers must make sure that press releases are carefully vetted before they are published, and not be overoptimistic about their results. And 'the limited information contained in press releases or abstracts should not be used alone to make treatment decisions or to implement changes in practice'. ${ }^{14}$

Breast cancer treatments have been the main topic of this essay, since this is my field of personal experience, but you may well think of other cancers where the media have misled readers, listeners and viewers. Patients need doctors who can distinguish between fact and spin, and help their patients to make genuinely informed decisions.

Competing interests None declared.

Acknowledgments I should like to acknowledge helpful comments from Dr Ann Johnson, Dr Charlotte Williamson, Co-ordinator of RAGE Jan Millington, and Professor Karol Sikora.

\section{REFERENCES}

1 Hanley B, Staley K. Yesterday's Women: the story of R.A.G.E. London: Macmillan Cancer Support, 2006

2 Owen JR, Ashton A, Bliss JM, et al. Effect of radiotherapy fraction size on tumour control in patients with early-stage breast cancer after local tumour excision: long-term results of a randomised trial. Lancet Oncol 2006;7:467-71

3 Cancer Research UK. Press release: Concentrated doses of radiotherapy shown to be better in treating breast cancer. London: Cancer Research UK, 29 May 2006

4 Yarnold J, Ashton A, Bliss J, et al. Fractionation sensitivity and dose response of late adverse effects in the breast after radiotherapy for early breast cancer: long-term results of a randomised trial. Radiother Oncol 2005; 75:9-17

5 Cuzick J, Stewart H, Rutqvist L, et al. Cause-specific mortality in longterm survivors of breast cancer who participated in trials of radiotherapy. J Clin Oncol 1994;12:447-53

6 Kurtz J, for the EUSOMA Working Party. The curative role of radiotherapy in the treatment of operable breast cancer. Eur $J$ Cancer 2002;38:1961-74

7 Sneeuw KCA, Aaronson NK, Yarnold JR, et al. Cosmetic and functional outcomes of breast conserving treatment for early stage breast cancer. 1. Comparison of patients' ratings, observers' ratings and objective assessments. Radiother Oncol 1992;25:153-9

8 Goodare H. Talking about tamoxifen [letter]. Health Expectations 2006; 9:91-4

9 Bagenal FS, Easton DF, Harris E, Chilvers CED, McElwain TJ Survival of patients with breast cancer attending Bristol Cancer Help Centre. Lancet 1990;336:606-10

10 Sherman J. Cancer patients at holistic centre 'are more likely to die'. The Times 6 September 1990

11 Cancer Research Campaign. Press release: Report on Alternative Cancer Therapy. London: Imperial Cancer Research Fund, 7 September 1990

12 Goodare H (ed.) Fighting spirit: the stories of women in the Bristol breast cancer survey. London: Scarlet Press, 1996

13 The ATAC (Arimidex, Tamoxifen Alone or in Combination) Trialists' Group. Anastrozole alone or in combination with tamoxifen versus tamoxifen alone for adjuvant treatment of postmenopausal women with early breast cancer: first results of the ATAC randomised trial. Lancet 2002;359:2131-9

14 Leading Edge. A STARing role for raloxifene? Lancet Oncol 2006;7:443 